\title{
A neuropsychiatric perspective on traumatic brain injury
}

\author{
Warren E. Lux, MD \\ Office of the Science Advisor, U.S. Environmental Protection Agency, Washington, DC; Center for Clinical Bioethics, \\ Georgetown University, Washington, DC
}

\begin{abstract}
Traumatic brain injury (TBI) due to closed mechanisms causes strain injuries to axons that increase in number and severity as injury severity increases. Axons that project up from the brain stem are vulnerable, even in milder concussive injuries, and include axons that participate in key monoaminergic pathways. Although called diffuse axonal injury, the supratentorial injury component typically shows an anterior preponderance in humans. As the injury forces increase, cerebral contusions may be superimposed on the axonal strain injuries, and these contusions show an anterior preponderance as well. The chronic neuropsychiatric manifestations of TBI reflect this injury distribution. In the cognitive sphere, these manifestations almost always include power function disturbances marked by difficulties with cognitive processing speed, multitasking, and cognitive endurance. These disturbances may then be followed by disturbances in executive function and selfawareness as injury severity increases. In the behavioral sphere, mood disturbances and disorders of behavioral control and regulation are particularly common.
\end{abstract}

Key words: behavior, cognition, depression, diffuse axonal injury, executive function, frontal lobes, mood, neuropsychiatry, rehabilitation, self-awareness, traumatic brain injury.

\section{INTRODUCTION}

Seen through the lens of the acute injury and its management, traumatic brain injury (TBI) would appear at first glance to be far removed from the concerns of what could properly be called neuropsychiatry. Rather, it might seem to be more a neurosurgical disorder or perhaps particularly relevant to conventional neurology or sports medicine. In the past 25 years, however, as acute management of both surgical and nonsurgical TBI has improved, impaired survivors have become increasingly common. And although these survivors face many different clinical issues, from epilepsy to motor problems to endocrine disorders, the TBI sequelae that affect their lives most profoundly are the chronic cognitive and behavioral problems that fall squarely within the domain of neuropsychiatry, a discipline that focuses on the relationship between the brain and its role in thinking, emotions, and behavior. Indeed, the paradigmatic historical patient for many of the core issues that now define neuropsychiatry was a young man from 19th century Vermont who survived a severe TBI.

During the summer of 1848, a 25-year-old railroad construction foreman named Phineas Gage was directing a blasting operation to clear a path for laying new track across Vermont for the Rutland \& Burlington Railroad. The operation went terribly awry, with the result that a pointed iron rod $3 \mathrm{ft} .7 \mathrm{in}$. in length, $11 / 4 \mathrm{in}$. in diameter, and $131 / 4 \mathrm{lb}$ in weight was propelled through Gage's face and head by an errant explosion. The rod entered

\footnotetext{
Abbreviations: $\mathrm{CT}=$ computed tomography, DAI $=$ diffuse axonal injury, $\mathrm{MRI}=$ magnetic resonance imaging, $\mathrm{TBI}=$ traumatic brain injury.

Address all correspondence to Warren E. Lux, MD; U.S. Environmental Protection Agency, 1200 Pennsylvania Ave, NW, Mail Code 8105R, Washington, DC 20460; 202-5643746; fax: 202-564-2070. Email: lux.warren@epa.gov

DOI: 10.1682/JRRD.2007.01.0009
} 
through his left cheek, continued up and through the base of his skull, traversed the anterior portions of his brain, and exited through the frontal bone at the top of his head. Remarkably, Gage survived both the wound and the subsequent infection with little in the way of adverse effects on his motor function, perception (save for the loss of his left eye), or intellect. Yet, as described in detail by Damasio on the basis of firsthand reports, Gage was an entirely different person after his physical recovery than he had been before [1]. Whereas previously he had been temperate, efficient, and capable, after the event he demonstrated aberrant social judgment, was unable to regulate his behavior normally, and could no longer use his various physical and intellectual strengths appropriately or effectively in the real world.

However, while the relevance of Gage's disorder to the newly emerging field of neuropsychiatry was becoming evident, traumatic patients of that sort were uncommon. Today, by contrast, TBI is one of the largest public health problems for children and young adults in the United States [2]. Consequently, no modern broad-based review of neuropsychiatry can be complete without addressing the problem of TBI. Yet, paradigmatic as his case was, Gage's injury differed from the most common injuries of today in one important respect: it was a penetrating injury. In penetrating injuries, of course, the region of the brain penetrated puts its particular stamp on the nature of the chronic cognitive and behavioral sequelae that develop. Today's injuries, by contrast, are predominantly closed. Gage just happened to injure an area of the brain that is particularly vulnerable to the effects of closed injury and so his is a dramatic, if fortuitous, exemplary case. But other vulnerabilities exist in closed injuries that need to be accounted for in expanding our understanding of TBI beyond that provided by Gage's injury, instructive as his case has been. A major purpose of this article is the dissection of those vulnerabilities and the placement of them in the context of the neuropsychiatric insights derived from Phineas Gage's case.

For several reasons then, I will focus on closed injuries in this article. First, they represent a major public health problem, as has already been pointed out, and understanding them provides clinicians with an important tool in addressing their neurocognitive and neurobehavioral consequences. More than that, however, I believe that knowledge of these injuries, their anatomic distribution, their probable neurochemical consequences, and the relationship of their neurobiology to the neuropsychiatric syndromes that develop provide a window into the brain that is compellingly illuminating, if still incomplete.

\section{PATHOPHYSIOLOGY OF TRAUMATIC BRAIN INJURY}

When the head is accelerated and decelerated abruptly in space, particularly when accompanied by a torsional head movement, strain forces are applied to nerve fibers (axons) throughout the brain [3-4]. The resulting axonal strain injuries, collectively referred to as diffuse axonal injury (DAI), represent the primary fundamental neuropathological change seen in TBI due to closed mechanisms regardless of injury severity, although the amount of DAI increases as injury severity increases [5]. Strictly speaking, if the head moves abruptly enough, as in whiplash injuries, it need not strike or be struck by another object for acceleration-deceleration phenomena sufficient to cause an axonal strain injury within the brain to occur [3], and clinicians who work with TBI are familiar with cases, usually mild, in which no head impact is involved. However, blunt-force trauma produces a particularly abrupt acceleration-deceleration and is, therefore, commonly a factor in these injuries. The most severe injuries tend to be caused by high-speed head impacts against immovable objects as in falls from height and motor vehicle accidents, in which victims either strike the windshield or are ejected from the vehicle [5].

Once an axonal strain injury occurs, by whatever mechanism, a number of consequences may ensue. First, axons may be torn apart completely, a phenomenon known as axotomy. These irreversible axonal shearing injuries may include gross ones that are immediately visible on neuroimaging studies. Delayed axotomy secondary to evolving pathological processes set off by the initial injury has also been well-demonstrated experimentally [6]. In addition, however, axons may clearly be damaged at a level that does not cause axotomy, although the axonal injury may cause alteration of electrophysiological function [4]. That such damage may be reversible is consistent with clinical observations of improvement after TBI and complete symptomatic recovery in many cases of mild TBI. Moreover, other aspects of neuronal physiology may be altered as well, theoretically either reversibly or irreversibly. Levels of chemical neurotransmitters contained in axons, for example, might be vulnerable to such alteration. One such class of agents is the 
monoaminergic neurotransmitters, and several studies suggest that they are indeed affected, both acutely and chronically [7-11]. Although the nature and distribution of the monoaminergic dysfunction following TBI remains to be more fully characterized, a number of clinical syndromes in which these chemical neurotransmitters have been implicated are common after TBI and will be discussed here.

Despite the fact that the axonal injuries described here are called diffuse, it may be something of a misnomer, because clearly, although they are multifocal and widespread, they are not uniformly distributed throughout the brain [4]. The clinical and experimental evidence cited by Ommaya and Gennarelli to support their centripetal theory suggests that the strain injury is maximal at the surfaces of the brain and extends inward to affect the diencephalic-mesencephalic core only as the injury becomes more severe [3]. However, as Gennarelli himself points out, this theory is only a first approximation; the exact distribution will be modified by the particulars of the individual traumatic event [4]. Moreover, the upper brain stem may be subjected to strains greater than would otherwise be expected because of the particular anatomy of that region, which is characterized by the relatively small attachment of the mesencephalon to the large masses of the cerebral hemispheres [4].

Superimposed on this modified centripetal distribution, at least in humans, appears to be an anterior-posterior or rostral-caudal gradient. Although little is written about this gradient in experimental animal models, clinical evidence in humans suggests that it is an important phenomenon. Aside from the neurobehavioral and neurocognitive correlates to be discussed here, the evidence from the neuroimaging literature strongly supports the existence of such a gradient, with frontal and anterior temporal lesions being significantly more frequent than lesions more posterior in the cerebral hemispheres [12-14]. Given the anatomy of the human brain and the membranes that invest it, perhaps this distribution is not surprising. Factors that could contribute to an anterior susceptibility to strain forces in humans include the relatively large mass of the human frontal lobes and the fact that the anterior human brain is less constrained in its movement inside the skull than is the posterior brain, which is embraced by the falx and the tentorium.

Although axonal strain may be the primary mechanism of neural injury in closed TBI, it is not the only one. As the injuries become more severe, cerebral contusions are increasingly seen, again with an anterior preponderance. The undersurfaces of the frontal lobes may also be abraded as they slide over the rough orbital surface of the frontal fossa. In addition, very diffuse secondary injuries can evolve and make their effects felt throughout the brain, including at sites far removed from the primary injury [15]. Nonetheless, in most patients, the broad pattern determined by the primary axonal strain injury and its neurochemical consequences will continue to be clinically apparent, sometimes reinforced by the orbital frontal abrasions and the anterior preponderance of the contusions that begin to accrue in the more severely injured patients. The neuropsychiatric syndromes that ensue, therefore, will be a consequence of this neurobiology and can be understood on that basis.

In summary then, persons who have sustained a closed TBI, whether mild, moderate, or severe, all sustain damage to axons and so end up with fewer axonal connections throughout their brains and/or less efficient electrophysiological function of at least some of the axonal connections that are present. Monoaminergic function is also likely impaired as a result of this injury in at least some patients. Both reversible and irreversible changes can occur, and when the injury is mild, complete symptomatic recovery is common. Even then, however, less reserve capacity appears to remain; the risk of persistent symptoms rises with repeat injuries. When the injury is moderate or severe, on the other hand, complete recovery is less common and at least some of the consequences of this axonal and/or neurochemical insufficiency are likely to be persistent. Nonetheless, when patients are symptomatic at all, we can predict a core set of impairments that patients with TBI will have in common, regardless of injury severity, and these impairments can be ascribed to the effects of sustaining any axonal strain sufficient to exceed the threshold for symptoms. Thereafter, as injury severity increases, we can also predict that the additional impairments that begin to accrue will arise from the greater degree of axonal damage and also from the anatomic distribution of both the axonal lesions and the other lesions, particularly the cerebral contusions and abrasions that mark greater injury severity. That distribution, as has been previously noted, shows an anterior-posterior gradient, so we should expect the neurocognitive and neurobehavioral consequences to reflect that. And finally, I should also note here that the old adage that "no two brain injuries are the same" is generally true, all the foregoing discussion notwithstanding. These are complex 
injuries, the exact patterns of which depend upon the particulars of both the injury and the person injured. The resultant heterogeneity, however, does not mean that underlying patterns and commonalities do not exist. The neuropathology presented here suggests that they do, and the relationship of that neuropathology to some of the classical neurocognitive and neurobehavioral syndromes commonly found after TBI is discussed in the following sections.

\section{IMPAIRMENT OF MENTAL POWER FUNCTION}

The most fundamental cognitive disturbance seen after TBI is impairment in what has been called power function, a term that refers to the basic capacity of the brain to engage efficiently in any cognitive task, regardless of what that task may be. The overall loss of mental "power" after TBI can be seen as a direct consequence of the widespread reduction in axonal performance resulting from DAI, even in its mildest form. The two critical components of power function are arousal and channel capacity; in the first instance, alteration in arousal at onset is characteristic of all but the mildest of TBIs. Once arousal recovers, however, the channel capacity disturbance makes itself clinically apparent. In a general sense, the term channel capacity refers to the number of channels available for processing information together with the amount of information that each channel can process. A computer or telephone network, for example, can have a high or a low channel capacity depending upon the number of connections in the network and the bandwidth characteristics of the materials from which the connections are made. The channel capacity disturbance that occurs in humans after TBI, then, has three particular characteristics that one might expect from such an analogy: slowed processing speed, difficulty with multitasking, and reduced cognitive endurance. This slowed cognitive processing speed is so characteristic of TBI that it can even be demonstrated in patients with extremely mild injuries who are asymptomatic [16]. Persons who are symptomatic, however, almost always have other symptoms related to the multitasking problem or the reduced cognitive endurance or both. In the cognitive domain, the difficulty with multitasking translates itself primarily into a difficulty in performing any cognitive task efficiently in a distracting environment. The initial treatment, then, is straightforward-limit stimulation and environmental distractions - and is part of the core management of most persons who have symptoms following TBI, from those who are mildly concussed to those with severe injuries and profound cognitive impairments. The multitasking problem, of course, has the potential to affect any cognitive function, and the complaint from the patient may reflect that. He or she may say, for example, "I can't concentrate" or "I'm having problems with my memory," yet show good (or at least better) memory and/ or attention in a stimulus-controlled environment. He or she simply cannot use his or her memory and attention systems effectively when subject to a multitasking requirement, even if no primary impairment is present in any of the specific components of either system. Of course, TBI often does affect primary components of attention and memory systems directly, particularly in the more severe injuries in which a greater burden of structural frontal and temporal damage exists. However, we must realize the primacy of the contribution of the disturbance in power function to many of the cognitive complaints that come from TBI survivors. A common feature of TBI that will become an increasing theme as we examine other deficits, particularly in the realm of executive function, is that TBI often affects the ability to use a function in the real world as much as or more than it affects the primary function itself. As a consequence, persons who have sustained a significant TBI can look strikingly normal in a quiet, structured clinic or even a neuropsychology laboratory, yet break down cognitively or behaviorally once they have to interact with the uncertainties and distractions of the real world.

The foregoing discussion of the effect of a multitasking deficit in the cognitive domain, however, is only one part of the story. We are also likely to see an effect, perhaps a significant one, in the behavioral domain. One of the behavioral consequences of being cognitively overwhelmed, even in nondisabled subjects, is irritability, and irritability is one of the common behavioral sequelae of TBI, whether mild, moderate, or severe. Again, I am not arguing here that all irritability after TBI arises from this mechanism. Certainly, other factors that will be discussed subsequently could cause irritability, including mood change and disorders of frontal regulation of behavioral output. However, I do believe that cognitive overload is a component in many patients, with support from the basic and widespread clinical observation that the same kind of stimulus-controlled environment that enhances cognition also tends to improve behavior. 
For the most part, the consequences of the impairments in cognitive endurance following TBI speak for themselves. Patients with symptomatic TBI are often simply unable to sustain a cognitive effort for as long as they could before their injury. The basic treatment is equally straightforward: introduce rest breaks. Potential pharmacological treatments have also been proposed for both this endurance problem and for the deficit in multitasking. These treatments generally revolve around psychostimulants or other drugs that enhance monoaminergic function, but no evidence-based consensus on drug treatment of this problem has yet been developed. One phenomenon that may be related to the observed reduction in cognitive endurance in many patients with TBI is the frequent complaint that life in general and cognitive tasks in particular require more effort. Recent functional magnetic resonance imaging (MRI) studies in patients with mild TBI may bear on this. In a study by McAllister et al., in particular, the experimental (mild TBI) group was able to perform as well on the cognitive task as the control (nondisabled) group but activated more cortex in doing so [17].

\section{IMPAIRMENT OF EXECUTIVE FUNCTION}

Mental power function is impaired in virtually all persons with TBI, regardless of injury severity, and that impairment may be the sole deficit in some cases of mild TBI. Once the injury becomes moderate or severe, however, and a greater burden of frontal injury is present, deficits in executive function begin to accrue with increasing frequency. The key components of executive function include anticipation, goal selection, planning, initiation, sequencing, monitoring (error detection), and self-correction (initiation of novel responses) [18]. The property that ties these components together is that they are all necessary for carrying out goal-directed activities successfully in unstructured real-world settings. Here, the anteriorposterior or rostral-caudal gradient of structural brain lesions in TBI becomes particularly relevant, as the critical neural substrate of these functions is the anterior forebrain. The dysexecutive syndromes that appear as the burden of frontal lesions increases, moreover, are as widely varied as the components of executive function itself. Some patients, for example, have great difficulty initiating any self-directed behaviors at all but are able to carry out most behaviors successfully once cued to initiate them. Others initiate easily and appear highly selfdirected but guide and regulate themselves poorly during the execution of behaviors for a variety of reasons, including impaired sequencing, poor error detection, cognitive inflexibility with failure to self-correct, and so forth. I should also note that these executive failures can, and often do, occur in TBI survivors whose basic intellectual functions are otherwise well-preserved because of relative sparing of posterior brain structures in the face of the previously mentioned anterior-posterior or rostralcaudal gradient. Thus, these patients can look much better in the structured clinic setting than they do when actually trying to guide themselves prospectively in the less structured and less predictable real world. The structure and expectations attendant upon being in the clinic, or in the neuropsychology laboratory, compensate at least in part for the executive deficits and thus enhance observed function. But once the patient returns to the real world, functions that were successfully executed in the structured setting begin to break down again. Memories, for example, that can be produced in a test setting where the patient knows what it is that he or she has to remember (and knows as well that he or she is supposed to be remembering them) can be difficult or impossible to retrieve in the real-world setting, where the executive task is to sort through one's memory bank de novo, inhibit nonrelevant memories, and retrieve only memories that are specifically related to the task at hand. One of my patients, who had one of these classic dysexecutive syndromes combined with the unusual feature of strikingly well-preserved insight, once put it thus: "You know, Dr. Lux," he said, "I'm just as smart as I always was. It's just that I can't use my smartness anymore."

\section{BEHAVIORAL DYSCONTROL}

If one considers the dysexecutive syndromes described previously as instances of lost or diminished cognitive regulation, an analogous problem can be identified in the behavioral sphere that is characterized by impaired social judgment and difficulty regulating emotional function as it contributes to and integrates with behavioral output. I do not wish to make too stark a contrast here, however, and I readily acknowledge that we are dealing with overlapping phenomena. Anticipation and goal selection, for example, are not purely "cognitive" functions and also require integration of emotional factors and social judgment. Yet 
some neuroanatomic differences exist that are worth noting. While lesions of the dorsolateral prefrontal convexity tend to be associated with the more purely cognitive executive disorders, disorders with a component of behavioral dyscontrol are correlated with ventromedial and orbital frontal lesions [19]. Phineas Gage, mentioned earlier in this article, had such a behavioral syndrome. And careful computer-aided mapping of his wound suggested significant damage to ventromedial frontal structures [20]. Patients with these lesions and syndromes can have long histories of recurrent chronic agitation and be quite difficult to manage. Their histories can include violence and aggression [19], and persons with such abnormalities may be disproportionately represented among violent criminals [21]. No widely agreed-upon pharmacological approach exists for managing behavioral abnormalities of this sort, and many different classes of psychoactive agents including antidepressants, antianxiety agents, antipsychotics, some anticonvulsants, and some antihypertensives, and others have been tried in clinical practice with varying degrees of success.

Yet all behavioral dyscontrol after TBI does not occur through this mechanism, and other behavioral dyscontrol syndromes must be carefully distinguished from those due to stable ventromedial frontal lesions for them to be managed appropriately. One such syndrome is the posttraumatic delirium that is seen during the early period of recovery, just after emergence from coma. One of the clinical hallmarks of this state is an extremely high degree of inattention, and the agitation that occurs is marked by this inattention and by the lability and disorganization that accompany it. Drugs that enhance serotonin and/or other monoamines and thereby improve attention are the rational choice in this state, in which central monoaminergic function is probably impaired. The use of some classes of antipsychotics and/or benzodiazepine antianxiety agents for this kind of agitation, unfortunately still widespread in some general medical settings, is rarely justified and may sometimes actually make the agitation paradoxically worse, presumably by adversely affecting the underlying inattention. This posttraumatic state is a transient, self-limited state that responds to stimulus control and the passage of time, although the agitation may merge clinically into agitation produced by one or more of the other mechanisms discussed here that are more chronic in nature. Thus, ongoing clinical vigilance and judgment are necessary to elucidate the likely mechanism of any agitation that is observed clinically so that management can be adjusted accordingly.

One important additional chronic behavioral condition that can develop after TBI, particularly in persons with known temporal lobe lesions, is the syndrome of episodic dyscontrol or intermittent explosive disorder. Patients with this syndrome are prone to rage attacks, i.e., brief outbursts of extreme anger, associated with agitated, aggressive, and/or violent behavior. The outbursts have a paroxysmal quality and may occur with little or no provocation. Epilepsy is a common comorbidity in these patients, although the rage attacks themselves are not felt to be epileptic. The condition has been reported to respond to the psychotropic anticonvulsants carbamazepine [22-23] and valproic acid [23].

\section{DISORDERS OF SELF-AWARENESS AND SELF- ASSESSMENT}

One of the most frustrating sequelae of moderate and severe TBI for caregivers, therapists, and families alike is the lack of insight that many patients exhibit into their deficits. This lack is usually not a problem after mild TBI; indeed, patients with mild TBI more often exhibit a hyperacute awareness of their problems. Rather, in most patients this lack of self-awareness appears to require a burden of frontal involvement not seen until the injury becomes moderate or severe. Once that threshold is surpassed, however, the subsequent loss of self-awareness and the associated inability of patients to assess their own capacities accurately complicates management enormously because it prevents the affected persons from adopting and using the very strategies that would compensate for their impaired functions, even when they possess all the other physical and mental tools to use these strategies well. Thus, this problem can be even more disabling for the patients than it is frustrating for their caregivers. It also highlights the "real world" nature of so many of the problems that persons with TBI experience. In a structured setting, cognitive prostheses can be introduced and maintained by others and the patient can thereby be protected from many of the consequences of lack of insight. But without close supervision, no such protection is available when an impaired person has to deal with novel, unexpected contingencies in the real world. One example of a simple cognitive prosthesis that might be introduced in a structured setting is a written 
and carefully sequenced "to do" list. When novel and unstructured real-world situations arise, however, either the list will not apply and the patient will be without a needed prosthesis or the list will apply but the patient will not have the insight to recognize that fact and use the list appropriately.

The nature of impaired self-awareness after TBI, moreover, is particularly interesting from a neuropsychiatric perspective. Not all neurologically based unawareness is frontal in origin; awareness of deficit can also be impaired by parietal, particularly right parietal, disease. In the parietal cases, patients appear unable to perceive their deficits. Because of the anterior-posterior, rostral-caudal gradient, however, unawareness syndromes are much more common in TBI on a frontal basis in the face of good preservation of parietal function. In these cases, the problem is generally not one of inability to perceive a deficit. Rather, the problem appears to be more a loss of the capacity to incorporate the knowledge of the deficit into one's global sense of oneself as an agent of action. Persons with this syndrome, therefore, can acknowledge a deficit that is demonstrated to them in a particular concrete situation. However, their subsequent behavior, verbal and otherwise, will not reflect an awareness of the deficit in any meaningful way, and they will say and do things as if they did not in fact have the deficit that they were earlier able to acknowledge [24]. In other words, the primary disturbance here is with one's ability to use available data about oneself to build an adequately accurate sense of who one is as a person not with the data themselves or the ability to acquire them in the first place.

\section{MOOD DISTURBANCES}

Alterations of mood are common after TBI [25]. They may be seen after injuries of any severity, and a full range of mood disturbances has been reported. The fact that ascending monoaminergic pathways are implicated in the basic neuropathology of TBI is probably critical to the high frequency of associated mood disorders, since these same neurotransmitters have been implicated in mood disorders associated with primary psychiatric diseases. Situational factors appear to play a role as well, however, and impaired social support is a commonly demonstrated risk factor for the development of mood disorder following TBI [25].
In clinical practice, unipolar depression is the most common mood disturbance encountered in survivors of TBI. The incidence of major depression varies considerably across studies, but the majority of studies suggest an overall incidence of somewhere between 25 and 50 percent [25-26]. All the various treatment options used for depression in primary psychiatric practice have also been suggested for use in depression following TBI, including serotonin reuptake inhibitors, mood-stabilizing anticonvulsants, psychostimulants, and electroconvulsive therapy [25]. The classical tricyclic antidepressants, however, probably have a lesser role here because of both the higher incidence of anticholinergic side-effects that can adversely affect cognition in persons who are already cognitively impaired and the suggestion, in at least some studies, of a relative treatment resistance to these agents [27-28]. Supportive psychotherapy, in addition to medication, can be used in patients whose cognitive status does not preclude it, including virtually all mildly injured patients, many moderately injured patients, and even some severely injured patients.

In addition to depression, both unipolar mania [29] and classical bipolar disorder [30] have been observed after TBI. The literature on these conditions is primarily in the form of case reports, and no large series, comprehensive incidence, or prevalence studies exist. Although these disorders undoubtedly occur, they appear to be uncommon and certainly do not present an overall clinical problem of the same degree of magnitude as that presented by post-TBI depression.

\section{PSYCHOSIS AND OTHER PSYCHIATRIC SYNDROMES}

Transient psychotic features can certainly appear early in recovery from coma during the posttraumatic delirium described earlier in the section on behavioral dyscontrol. This state, however, is dominated by confusion, gross inattention, and extreme cerebral disorganization and the psychotic fragments are incorporated into this context. Far more clinically problematic, however, is the more persistent, late-onset psychosis with delusions, hallucinations, and other psychotic symptoms that may occur in a delayed fashion following TBI [31]. Gualtieri regards the effect of this psychosis on the human personality as the most severe and devastating outcome of TBI [32]. Neither the incidence nor prevalence of delayed or 
late-onset psychosis following TBI is known. In my experience, it is uncommon. Gualtieri, on the other hand, believes that it could have a prevalence rate as high as that of posttraumatic epilepsy [32].

A number of other psychiatric syndromes following TBI have been reported, particularly ones in which frontal and temporal structures and monoaminergic function have been implicated. Obsessive-compulsive disorder has been particularly well characterized [33-34]. Panic disorder following TBI has also been described [35].

\section{ASSESSMENT AND TREATMENT CONSIDERATIONS}

History is particularly important in the clinical assessment of persons with TBI. First, patients with TBI will necessarily display their cognition and behavior as they relate their history, and the way in which they do so may be as instructive as the results of more formal assessments, as important as the latter are. Moreover, history gives one a handle on function in the real world, something that is crucial in patients with TBI because of the frequent dissociation between function in structured, stimulus-controlled settings and function in dynamic, novel, demanding situations. Because of the possibility of lack of insight and impaired self-assessment, however, history from persons other than the patient is necessary in many cases. In a related way, observation of patients in real-world settings by therapists or others can also be an important component of any complete assessment of a patient with TBI.

The foregoing principles notwithstanding, formal evaluation of patients with TBI in the clinic setting is essential and should include a complete neurological and mental status examination. A comprehensive neuropsychological assessment is also an important component of the evaluation of any patient with TBI who is persistently symptomatic, and neuropsychological testing gives a level of detail in a standardized, validated way that is impossible to obtain through any other means. More recently, computer-aided repeated measures cognitive test batteries have been developed that appear to be quite sensitive to the effects of even mild TBI [36]. Lack of specificity and variation among and within subjects, however, are problems that remain to be resolved, so any use of these instruments needs to be thoughtfully incorporated into a careful, multilevel, overall clinical assessment.
If a patient with TBI requires a neuroimaging study because of injury severity, examination findings, progression or persistence of symptoms, or other cause, MRI is significantly more sensitive than computed tomography (CT) in demonstrating traumatic lesions [12-14]. In cases where an acute surgical lesion is suspected, the speed and ease of use of CT and its adequate sensitivity for such lesions make it the primary imaging modality. CT is also used as the primary study in patients who are precluded from being exposed to an MRI for one of several clinical reasons. However, when it can be used safely, MRI provides superior information about the nature, extent, and distribution of traumatic lesions.

Treatment options for the neurocognitive and neurobehavioral sequelae of TBI are varied. Rehabilitation interventions play a role, particularly in the more severely injured individuals [37-40], but much work needs to be performed to further define that role. In more mildly injured patients, a simple educational intervention significantly enhances symptomatic recovery [41].

For many of the conditions discussed in this article, the inclusion of medications in the treatment regimen has been proposed with varying levels of evidence and justification. A complete review of cognitive and behavioral pharmacology following TBI is beyond the scope of this article, and the reader is referred to one of the several reviews that currently exist [42-45]. Clearly, however, the various neurotransmitter alterations that can be produced by TBI offer opportunities for targeted pharmacological interventions that might benefit at least some components of the cognitive and behavioral syndromes that develop.

And finally, we must remember that TBI needs to be understood as a condition that affects not just an individual but also the individual's interaction with all other persons and things in his or her environment. Thus, treatment should reflect the environment as well. Alteration of the environment to make it more structured and stimuluscontrolled, for example, may be as important as alteration of anything within the patient. And education, counseling, and other treatment interventions for those who are closely involved with the patient in his or her day-to-day life may be critical to a successful therapeutic outcome.

\section{DISCUSSION AND CONCLUSIONS}

TBI is the most common cause of death and disability in children and young adults in the United States [2]. 
A normally functioning brain is critical to many human functions but is particularly relevant to human thought, awareness, emotion, and behavior. Injuries to the brain, therefore, often disrupt these functions and commonly result in deficits that fall within the domain of neuropsychiatry. As a consequence, understanding TBI and the interventions that mitigate the resulting deficits requires approaching TBI from a neuropsychiatric perspective. This review attempts to link the known neuropathological features of TBI with the neuropsychiatric consequences and, in turn, with the therapeutic interventions that logically follow. When treating clinicians, family and friends, and sometimes the patients themselves make these links, they acquire a deeper understanding of TBI and so are able to manage the resulting neuropsychiatric deficits more effectively. Yet the benefits of this approach go even further and transcend the clinical domain, as the links that are made also provide valuable insights, however rudimentary, into how the brain functions as the organ of the mind.

\section{ACKNOWLEDGMENTS}

An earlier draft of this work was developed while the author was at the Defense and Veterans Brain Injury Center in Washington, DC. The support of the Center and especially of the Center's Director, Dr. Deborah Warden, is gratefully acknowledged.

The views expressed in this article are solely those of the author and do not reflect official positions or policies of the U.S. Environmental Protection Agency, the Department of Defense, or the U.S. Government.

This material was unfunded at the time of manuscript preparation.

The author has declared that no competing interests exist.

\section{REFERENCES}

1. Damasio AR. Unpleasantness in Vermont. In: Descartes' error: Emotion, reason, and the human brain. New York (NY): Putnam; 1994.

2. Thurman DJ, National Center for Injury Prevention and Control, U.S. Division of Acute Care, Rehabilitation Research, and Disability Prevention. Traumatic brain injury in the United States: A report to congress. Atlanta (GA): Centers for Disease Control and Prevention; 1999.
3. Ommaya AK, Gennarelli TA. Cerebral concussion and traumatic unconsciousness. Correlation of experimental and clinical observations of blunt head injuries. Brain. 1974; 97(4):633-54. [PMID: 4215541]

4. Gennarelli TA. Mechanisms and pathophysiology of cerebral concussion. J Head Trauma Rehabil. 1986;1(2):23-29.

5. Alexander MP. Mild traumatic brain injury: Pathophysiology, natural history, and clinical management. Neurology. 1995;45(7):1253-60. [PMID: 7617178]

6. Povlishock JT. Pathobiology of traumatically induced axonal injury in animals and man. Ann Emerg Med. 1993; 22(6):980-86. [PMID: 8503536]

7. Bareggi SR, Porta M, Selenati A, Assael BM, Calderini G, Collice M, Rossanda M, Morselli PL. Homovanillic acid and 5-hydroxyindole-acetic acid in the CSF of patients after a severe head injury: I. Lumbar CSF concentration in chronic brain post-traumatic syndromes. Eur Neurol. 1975; 13(6):528-44. [PMID: 1193100]

8. Porta M, Bareggi SR, Collice M, Assael BM, Selenati A, Calderini G, Rossanda M, Morselli PL. Homovanillic acid and 5-hydroxyindole-acetic acid in the CSF of patients after a severe head injury. II. Ventricular CSF concentrations in acute brain post-traumatic syndromes. Eur Neurol. 1975;13(6):545-54. [PMID: 1193101]

9. Vecht CJ, Van Woerkom TC, Teelken AW, Minderhoud JM. 5-hydroxyindoleacetic acid (5-HIAA) levels in the cerebrospinal fluid in consciousness and unconsciousness after head injury. Life Sci. 1975;16(7):1179-85.

[PMID: 1134190]

10. Vecht CJ, Van Woerkom CA, Teelken AW, Minderhoud JM. Homovanillic acid and 5-hydroxyindoleacetic acid cerebrospinal fluid levels. A study with and without probenecid administration of their relationship to the state of consciousness after head injury. Arch Neurol. 1975; 32(12):792-97. [PMID: 1203031]

11. Van Woerkom TC, Teelken AW, Minderhous JM. Difference in neurotransmitter metabolism in frontotemporallobe contusion and diffuse cerebral contusion. Lancet. 1977; 1(8015):812-13. [PMID: 66617]

12. Levin HS, Williams D, Crofford MJ, High WM Jr, Eisenberg HM, Amparo EG, Guinto FC Jr, Kalisky Z, Handel SF, Goldman AM. Relationship of depth of brain lesions to consciousness and outcome after closed head injury. J Neurosurg. 1988;69(6):861-66. [PMID: 3193191]

13. Levin HS, Mendelsohn D, Lilly MA, Yeakley J, Song J, Scheibel RS, Harward H, Fletcher JM, Kufera JA, Davidson $\mathrm{KC}$, Bruce D. Magnetic resonance imaging in relation to functional outcome of pediatric closed head injury: A test of the Ommaya-Gennarelli model. Neurosurgery. 1997; 40(3):432-41. [PMID: 9055281]

14. Van der Naalt J, Hew JM, Van Zomeren AH, Sluiter WJ, Minderhoud JM. Computed tomography and magnetic resonance imaging in mild to moderate head injury: Early and 
late imaging related to outcome. Ann Neurol. 1999;46(1): 70-78. [PMID: 10401782]

15. Wu HM, Huang SC, Hattori N, Glenn TC, Vespa PM, Hovda DA, Bergsneider M. Subcortical white matter metabolic changes remote from focal hemorrhagic lesions suggest diffuse injury after human traumatic brain injury. Neurosurgery. 2004;55(6):1306-17. [PMID: 15574212]

16. Warden DL, Bleiberg J, Cameron KL, Ecklund J, Walter J, Sparling MB, Reeves D, Reynolds KY, Arciero R. Persistent prolongation of simple reaction time in sports concussion. Neurology. 2001;57(3):524-26. [PMID: 11502926]

17. McAllister TW, Saykin AJ, Flashman LA, Sparling MB, Johnson SC, Guerin SJ, Mamourian AC, Weaver JB, Yanofsky N. Brain activation during working memory 1 month after mild traumatic brain injury: A functional MRI study. Neurology. 1999;53(6):1300-1308.

[PMID: 10522888]

18. Lezak MD. Assessment of psychosocial dysfunctions resulting from head trauma. In: Lezak MD, editor. Assessment of the behavioral consequences of head trauma. New York (NY): A. R. Liss; 1989. p. 113-43.

19. Grafman J, Schwab K, Warden D, Pridgen A, Brown HR, Salazar AM. Frontal lobe injuries, violence, and aggression: A report of the Vietnam Head Injury Study. Neurology. 1996;46(5):1231-38. [PMID: 8628458]

20. Damasio H, Grabowski T, Frank R, Galaburda AM, Damasio AR. The return of Phineas Gage: Clues about the brain from the skull of a famous patient. Science. 1994;264(5162): 1102-5. [PMID: 8178168] Erratum in: Science. 1994; 265(5176):1159.

21. Blake PY, Pincus JH, Buckner C. Neurologic abnormalities in murderers. Neurology. 1995;45(9):1641-47. [PMID: 7675220]

22. Lewin J, Sumners D. Successful treatment of episodic dyscontrol with carbamazepine. Br J Psychiatry. 1992;161: 261-62. [PMID: 1521112]

23. Gualtieri CT. Intermittent explosive disorder. In: Neuropsychiatry and behavioral pharmacology. New York (NY): Springer-Verlag; 1991. p. 109-10.

24. Anderson J, Lux W. Knowing your own strength: Accurate self-assessment as a requirement for personal autonomy. Philos Psychiatry Psychol. 2004;11(4):279-94.

25. Jorge R, Robinson RG. Mood disorders following traumatic brain injury. Int Rev Psychiatry. 2003;15(4):317-27. [PMID: 15276953]

26. Fedoroff JP, Starkstein SE, Forrester AW, Geisler FH, Jorge RE, Arndt SV, Robinson RG. Depression in patients with acute traumatic brain injury. Am J Psychiatry. 1992;149(7): 918-23. [PMID: 1609872]

27. Dinan TG, Mobayed M. Treatment resistance of depression after head injury: A preliminary study of amitriptyline response. Acta Psychiatr Scand. 1992;85(4):292-94. [PMID: 1595364]

28. Saran AS. Depression after minor closed head injury: Role of dexamethasone suppression test and antidepressants. J Clin Psychiatry. 1985;46(8):335-38. [PMID: 4019422]

29. Bakchine S, Lacomblez L, Benoit N, Parisot D, Chain F, Lhermitte F. Manic-like state after bilateral orbitofrontal and right temporoparietal injury: Efficacy of clonidine. Neurology. 1989;39(6):777-81. [PMID: 2725871]

30. Pope HG Jr, McElroy SL, Satlin A, Hudson JI, Keck PE Jr, Kalish R. Head injury, bipolar disorder, and response to valproate. Comp Psychiatry. 1988;29(1):34-38. [PMID: 3125002$]$

31. Fujii DE, Ahmed I. Psychosis secondary to traumatic brain injury. Neuropsychiatry Neuropsychol Behav Neurol. 1996; 9(2):133-38.

32. Gualtieri CT. Psychosis. In: Neuropsychiatry and behavioral pharmacology. New York (NY): Springer-Verlag; 1991. p. 34-35.

33. Berthier ML, Kulisevsky J, Gironell A, Heras JA. Obsessive-compulsive disorder associated with brain lesions: Clinical phenomenology, cognitive function, and anatomic correlates. Neurology. 1996;47(2):353-61. [PMID: 8757004]

34. Hollander E, Wong CM. The relationship between executive function impairment and serotonergic sensitivity in obsessive-compulsive disorder. Neuropsychiatry Neuropsychol Behav Neurol. 1996;9(4):230-33.

35. Scheutzow MH, Wiercisiewski DR. Panic disorder in a patient with traumatic brain injury: A case report and discussion. Brain Inj. 1999;13(9):705-14. [PMID: 10507452]

36. Bleiberg J, Cernich AN, Cameron K, Sun W, Peck K, Ecklund PJ, Reeves D, Uhorchak J, Sparling MB, Warden DL. Duration of cognitive impairment after sports concussion. Neurosurgery. 2004;54(5):1073-80. [PMID: 15113460]

37. Cope DN, Hall K. Head injury rehabilitation: Benefit of early intervention. Arch Phys Med Rehabil. 1982;63(9): 433-37. [PMID: 7115044]

38. Mills VM, Nesbeda T, Katz DI, Alexander MP. Outcomes for traumatically brain-injured patients following postacute rehabilitation programmes. Brain Inj. 1992;6(3):219-28. [PMID: 1581745]

39. Cope DN. The effectiveness of traumatic brain injury rehabilitation: A review. Brain Inj. 1995;9(7):649-70. [PMID: 8680395]

40. Salazar AM, Warden DL, Schwab K, Spector J, Braverman S, Walter J, Cole R, Rosner MM, Martin EM, Ecklund J, Ellenbogen RG. Cognitive rehabilitation for traumatic brain injury: A randomized trial. Defense and Veterans Head Injury Program (DVHIP) Study Group. JAMA. 2000; 283(23):3075-81. [PMID: 10865301]

41. Ponsford J, Willmott C, Rothwell A, Cameron P, Kelly AM, Nelms R, Curran C. Impact of early intervention on 
outcome following mild head injury in adults. J Neurol Neurosurg Psychiatry. 2002;73(3):330-32.

[PMID: 12185174$]$

42. Gualtieri CT. Pharmacotherapy and the neurobehavioral sequelae of traumatic brain injury. Brain Inj. 1988;2(2): 101-29. [PMID: 3048503]

43. Gualtieri CT. The psychopharmacology of traumatic brain injury. In: Neuropsychiatry and behavioral pharmacology. New York (NY): Springer-Verlag; 1991. p. 37-88.

44. Lux WE. Pharmacological strategies in the management of cognition and behavior following traumatic brain injury. In:
Uzzell BP, Stonnington HH, editors. Recovery after traumatic brain injury. Mahwah (NJ): Lawrence Erlbaum Associates; 1996. p. 99-112.

45. Lee HB, Lyketsos CG, Rao V. Pharmacological management of the psychiatric aspects of traumatic brain injury. Int Rev Psychiatry. 2003;15(4):359-70.

[PMID: 15276957]

Submitted for publication January 22, 2007. Accepted in revised form June 21, 2007. 